\title{
Multi-parameter, impulsive effects and positive periodic solutions of first-order functional differential equations
}

\author{
Xuemei Zhang ${ }^{1 *}$ and Meiqiang Feng ${ }^{2}$
}

${ }^{*}$ Correspondence: zxm74@sina.com
${ }^{1}$ Department of Mathematics and
Physics, North China Electric Power
University, Beijing, 102206, People's
Republic of China
Full list of author information is
available at the end of the article

\begin{abstract}
The authors consider the existence of positive periodic solution for the impulsive functional differential equation $u^{\prime}(t)=-a(t) g\left(u\left(h_{1}(t)\right)\right) u(t)+\lambda b(t) f\left(t, u\left(h_{2}(t)\right), \int_{-\zeta}^{0} e(s) u(t+s) d s\right), t \in \mathbf{R}, t \neq t_{k}$, $u\left(t_{k}^{+}\right)-u\left(t_{k}\right)=\mu l_{k}\left(t_{k}, u\left(t_{k}\right)\right), k \in \mathbf{Z}$, where $\lambda>0$ and $\mu>0$ are two parameters. Several new and more general existence and multiplicity results are derived in terms of different values of $\lambda>0$ and $\mu>0$. Here we not only consider the case that $g$ is bounded, but the case that $g$ is not necessarily bounded is also considered. Our results improve those in (Ma et al. in J. Math. Anal. Appl. 384:527-535, 2011; Li et al. in Comput. Math. Appl. 56:2556-2560, 2008). Moreover, the parameter dependence of the periodic solution is also studied.
\end{abstract}

Keywords: multi-parameter; impulsive effect; positive periodic solution; functional differential equations; parameter dependence of periodic solution

\section{Introduction}

Impulsive functional differential equations are emerging as an important area of investigation, since various real-world simulation processes which depend on their prehistory and are subject to short time disturbances, such as population dynamics, biology, biotechnology, industrial robotic, pharmacokinetics, optimal control, etc., can be expressed by functional differential equations with impulses; see [1-5]. Moreover, Cushing [6] pointed out that any biological or environmental parameters are naturally subject to fluctuations in time, due to seasonal effects of weather, food supply, mating habits, hunting or harvesting seasons, etc. Therefore, it is necessary and important to consider models with periodic ecological parameters or perturbations, which may be more natural (see [7-27]). For example, Liu and Li [9] considered the following first-order functional differential equation with a parameter:

$$
u^{\prime}(t)=-a(t) u(t)+\lambda b(t) f\left(u\left(t-\tau_{0}(t)\right)\right)
$$

By using the eigenvalue theory of operators, the authors established several sufficient conditions for the existence of positive $T$-periodic solutions.

(c) 2015 Zhang and Feng. This article is distributed under the terms of the Creative Commons Attribution 4.0 International License (http://creativecommons.org/licenses/by/4.0/), which permits unrestricted use, distribution, and reproduction in any medium, provided you give appropriate credit to the original author(s) and the source, provide a link to the Creative Commons license, and indicate if changes were made. 
In [10], Wang investigated the following first-order functional differential equation with a parameter:

$$
u^{\prime}(t)=a(t) g(u(t)) u(t)-\lambda b(t) f\left(u\left(t-\tau_{0}(t)\right)\right)
$$

and by virtue of the fixed point theorems in a cone, the author obtained the existence, non-existence, and multiplicity of positive $\omega$-periodic solutions under suitable conditions imposed on the nonlinear $\operatorname{term} f$.

Yan [28] pointed out that (A) and (B) include various models. For example, Cooke and Kaplan [29], and Cooke and Yorke [30] studied, respectively, the equation

$$
u^{\prime}(t)=-g(y(t))+f(y(t-\tau))
$$

for epidemic and population growth and gonorrhea epidemics.

Mackey and Glass [31] considered the following equation:

$$
u^{\prime}(t)=-\gamma u(t)+\frac{\beta_{0} \theta^{n}}{\theta^{n}+u^{n}(t-\tau)}
$$

for model of hematopoiesis (blood cell production).

For some other recent excellent results and applications on (A) and (B), we refer the reader to papers by Jin and Wang [32], Ye et al. [33], Li et al. [34], Graef and Kong [35, 36], Franco et al. [37], Wu and Wang [38], Padhi and Srivastava [39], Olach [40], Amster and Idels [41], Chen et al. [42], Ma et al. [43] and Weng and Sun [44].

As regards the ideal of an impulsive perturbation, in [28], a pioneer paper concerning the solvability of periodic problem, Yan considered the following impulsive functional differential equation:

$$
\begin{cases}u^{\prime}(t)=g(t, u(t))-\lambda f\left(t, u\left(t-\tau_{0}(t)\right)\right), & t \in \mathbf{R}, t \neq t_{k} \\ u\left(t_{k}^{+}\right)-u\left(t_{k}\right)=\mu I_{k}\left(t_{k}, u\left(t_{k}-\tau\left(t_{k}\right)\right)\right), & k \in \mathbf{Z} .\end{cases}
$$

By utilizing a well-known fixed point index theorem in cone, the author got some sufficient conditions of the existence and non-existence of positive periodic solutions of (C).

In this paper, we investigate the existence of positive periodic solutions of the impulsive functional differential equation

$$
\left\{\begin{aligned}
& u^{\prime}(t)=-a(t) g\left(u\left(h_{1}(t)\right)\right) u(t) \\
&+\lambda b(t) f\left(t, u\left(h_{2}(t)\right), \int_{-\zeta}^{0} e(s) u(t+s) d s\right), \quad t \in \mathbf{R}, t \neq t_{k}, \\
& u\left(t_{k}^{+}\right)-u\left(t_{k}\right)=\mu I_{k}\left(t_{k}, u\left(t_{k}\right)\right), \quad k \in \mathbf{Z},
\end{aligned}\right.
$$

where $\lambda>0$ and $\mu>0$ are parameters, $e \in C([-\zeta, 0], \mathbf{R}), \int_{-\zeta}^{0} e(s) d s=1$, and $\left\{t_{k}, k \in \mathbf{Z}\right\}$ is an increasing sequence of real number with $\lim _{k \rightarrow \pm \infty} t_{k}= \pm \infty$. In addition, let

$$
\omega>0, \quad \mathbf{R}=(-\infty,+\infty), \quad \mathbf{R}_{+}=[0,+\infty)
$$

We make the following hypotheses:

$\left(\mathrm{H}_{1}\right) a, b \in C\left(\mathbf{R}, \mathbf{R}_{+}\right)$are $\omega$-periodic functions satisfying $\int_{0}^{\omega} a(t) d t>0, \int_{0}^{\omega} b(t) d t>0 ;$ 
$\left(\mathrm{H}_{2}\right) h_{i} \in C\left(\mathbf{R}, \mathbf{R}_{+}\right)(i=1,2)$ is $\omega$-periodic function, $g \in C\left(\mathbf{R}_{+}, \mathbf{R}_{+}\right)$;

$\left(\mathrm{H}_{3}\right) f \in C\left(\mathbf{R} \times \mathbf{R}_{+} \times \mathbf{R}_{+}, \mathbf{R}_{+}\right)$is $\omega$-periodic function in $t$; moreover, $f(t, x, y)>0$ for all $t, x>0$ and $y>0$

$\left(\mathrm{H}_{4}\right) \quad I_{k} \in C\left(\mathbf{R} \times \mathbf{R}_{+}, \mathbf{R}_{+}\right)$is $\omega$-periodic function in $t$ and $I_{k}(t, x)>0$ for all $t$ and $x>0, k \in \mathbf{Z}$; moreover, there exists a positive integer $p$ such that

$$
I_{k+p}\left(t_{k+p}, x\right)=I_{k}\left(t_{k}, x\right), \quad t_{k+p}=t_{k}+\omega, k \in \mathbf{Z}
$$

Without loss of generality, we assume that $[0, \omega] \cap\left\{t_{k}, k \in \mathbf{Z}\right\}=\left\{t_{0}, t_{1}, \ldots, t_{p-1}\right\}$.

Some special cases of (1.1) have been investigated. For example, Li et al. [34] discussed problem (1.1) in the case that $\mu \equiv 1$ and $I_{k}(t, u)=I_{k}(u), k \in \mathbf{Z}^{+}$. By using the well-known Leggett-Williams multiple fixed point theorem, the authors obtained the existence of multiple positive periodic solutions of problem (1.1). However, in [34], Li et al. only dealt with the case that there exist two positive constants $l$ and $L$ such that

$$
0<l \leq g(u) \leq L<\infty \quad \text { for all } u>0 .
$$

It is interesting to know whether there is a positive periodic solution of (1.1) when $g$ does not satisfy (1.2). Very recently, Ma et al. [43] studied the existence of positive periodic solutions of (1.1) under the following two cases:

$$
\begin{aligned}
& 0<l \leq g(u)<\infty \text { for all } u>0, \\
& 0<g(u) \leq L \text { for all } u>0,
\end{aligned}
$$

where $l$ and $L$ are two positive constants. However, Ma et al. obtained the existence of at least one positive periodic solution only for $I_{k}(t, u) \equiv 0, k \in \mathbf{Z}$.

At the same time, we notice that there is almost no paper except [28] studying impulsive functional differential equations with two parameters. By using a well-known fixed point index theorem due to Krasnoselskii, the author obtained the following results in the case $g$ is bounded and $e(t) \equiv 0, t \in[-\zeta, 0]$.

Theorem A (Theorem 3.1 of [28]) Let the following conditions hold.

$\left(\mathrm{A}_{1}\right) \lambda>0$ and $\mu \geq 0$ are parameters.

$\left(\mathrm{A}_{2}\right) t_{k}, k \in \mathbf{Z}$, is an increasing sequence of real number with $\lim _{k \rightarrow \pm \infty} t_{k}= \pm \infty$.

$\left(\mathrm{A}_{3}\right)$ h and $f: \mathbf{R} \times \mathbf{R}^{+} \rightarrow \mathbf{R}^{+}$satisfy Carathéodory conditions, that is, $h(t, y)$ and $f(t, y)$ are locally Lebesgue measurable in $t$ for each fixed $y$ and are continuous in $y$ for each fixed $t$, are $\omega$-periodic functions in $t$. Moreover, $f(t, y)>0$ for all $t$ and $y>0 . \tau: \mathbf{R} \rightarrow \mathbf{R}$ is locally bounded Lebesgue measurable $\omega$-periodic function.

$\left(\mathrm{A}_{4}\right)$ There exist $\omega$-periodic functions $a_{1}$ and $a_{2}: \mathbf{R} \rightarrow \mathbf{R}^{+}$which are locally bounded Lebesgue measurable so that $a_{1}(t) y \leq h(t, y) \leq a_{2}(t) y$ for all $y>0$ and $\lim _{y \rightarrow 0^{+}} h(t, y) y$ exists, $\int_{0}^{\omega} a_{1}(t) d t>0$.

(A5) $I_{k}: \mathbf{R} \times[0, \infty) \rightarrow \mathbf{R}, k \in Z$, satisfy Carathéodory conditions and are $\omega$-periodic functions in $t$ and there exists an integer $\rho$ such that $I_{k+\rho}\left(t_{k+\rho}, y\right)=I_{k}\left(t_{k}, y\right), t_{k+\rho}=t_{k}+\omega$, $k \in \mathbf{Z}$. Moreover, $I_{k}(t, 0)=0$ for all $k \in \mathbf{Z}$.

( $\left.\mathrm{A}_{6}\right)$ p and $q: \mathbf{R} \rightarrow \mathbf{R}^{+}$are positive bounded Lebesgue measurable $\omega$-periodic functions and is bounded away from zero. 
Suppose further that $f^{0}, f_{\infty}, I_{\infty}$, and $I^{0}$ are positive constants, where

$$
\begin{aligned}
& f^{0}=\limsup _{y \rightarrow 0^{+}} \max _{t \in[0, \omega]} \frac{f(t, y)}{p(t) y}, \quad f_{\infty}=\liminf _{y \rightarrow \infty} \min _{t \in[0, \omega]} \frac{f(t, y)}{p(t) y}, \\
& I^{0}=\limsup _{y \rightarrow 0^{+}} \max _{t \in[0, \omega], k \in[0, \rho]} \frac{I_{k}(t, y)}{q(t) y}, \quad I_{\infty}=\liminf _{y \rightarrow \infty} \min _{t \in[0, \omega], k \in[0, \rho]} \frac{I_{k}(t, y)}{q(t) y} .
\end{aligned}
$$

If

$$
\beta\left(\lambda f^{0} P+\mu I^{0} Q\right)<1
$$

and

$$
\alpha \sigma\left(\lambda f_{\infty} P+\mu I_{\infty} Q\right)>1
$$

then problem (1.1) has a positive $\omega$-periodic solution.

It is not difficult to see that the conditions of Theorem A are not the optimal conditions which guarantee the existence of at least one positive periodic solution for problem (1.1). In fact, if

$$
\beta\left(\lambda f^{0} P+\mu I^{0} Q\right)<1
$$

or

$$
\alpha \sigma\left(\lambda f_{\infty} P+\mu I_{\infty} Q\right)>1
$$

we can prove that problem (1.1) has at least one positive periodic solution, respectively.

Motivated by the papers mentioned above, we will extend the results of $[9,10,28,32,34$, 43] to problem (1.1) in the case that $g$ satisfies (1.2), (1.3), and (1.4), respectively. Moreover, the parameter dependence of the periodic solution is also studied.

The rest of the paper is organized as follows. In Section 2, we study the existence and multiplicity results of positive periodic solutions for problem (1.1) when the function $g$ satisfies (1.2). In Section 3, we establish the dependence results of the positive periodic solution on the parameter for problem (1.1). The final section of the paper studies the existence of positive periodic solutions for problem (1.1) when the function $g$ satisfies (1.3) or (1.4). Some remarks are also given to explain why we do not consider the multiplicity of positive periodic solutions and the dependence results of the positive periodic solution on the parameter when the function $g$ satisfies (1.3) or (1.4).

\section{Existence results under (1.2)}

\subsection{Main results}

In this section, we state the main results, including existence and multiplicity results of positive periodic solutions for problem (1.1).

For convenience, we introduce the following notations:

$$
\begin{array}{ll}
f^{0}=\limsup _{x \rightarrow 0^{+}, y \rightarrow 0^{+}} \max _{t \in J} \frac{f(t, x, y)}{x}, & f^{\infty}=\limsup _{x \rightarrow \infty, y \rightarrow \infty} \max _{t \in J} \frac{f(t, x, y)}{x}, \\
f_{0}=\liminf _{x \rightarrow 0^{+}, y \rightarrow 0^{+}} \min _{t \in J} \frac{f(t, x, y)}{x}, & f_{\infty}=\liminf _{x \rightarrow \infty, y \rightarrow \infty} \min _{t \in J} \frac{f(t, x, y)}{x},
\end{array}
$$




$$
\begin{array}{ll}
I^{0}(k)=\limsup _{x \rightarrow 0^{+}} \frac{I_{k}\left(t_{k}, x\right)}{x}, & I^{\infty}(k)=\limsup _{x \rightarrow \infty} \frac{I_{k}\left(t_{k}, x\right)}{x}, \\
I_{0}(k)=\liminf _{x \rightarrow 0^{+}} \frac{I_{k}\left(t_{k}, x\right)}{x}, & I_{\infty}(k)=\liminf _{x \rightarrow \infty} \frac{I_{k}\left(t_{k}, x\right)}{x},
\end{array}
$$

where $J=[0, \omega], k=0,1, \ldots, p-1$. Moreover, we choose four numbers $r, r_{1}, r_{2}$, and $R$ satisfying

$$
0<r<r_{1}<\delta r_{2}<r_{2}<R<+\infty,
$$

where $\delta$ is defined in (2.9).

Theorem 2.1 Let $\left(\mathrm{H}_{1}\right)-\left(\mathrm{H}_{4}\right)$ hold. Assume that $0<f_{\infty} \leq f^{\infty}<+\infty, 0<I_{\infty}(k) \leq I^{\infty}(k)<$ $+\infty, k=0,1, \ldots, p-1$, and the function $g$ satisfies (1.2). Then

(i) there exist $\lambda_{0}>0$ and $\mu_{0}>0$ such that, for any $\lambda>\lambda_{0}$ and $\mu>\mu_{0}$, problem (1.1) has a positive periodic solution $u(t)$ with

$$
\delta r \leq u(t) \leq \frac{1}{\delta} R, \quad t \in J
$$

(ii) there exist $\bar{\lambda}_{0}>0$ and $\bar{\mu}_{0}>0$ such that, for any $0<\lambda<\bar{\lambda}_{0}$ and $0<\mu<\bar{\mu}_{0}$, problem

(1.1) has a positive periodic solution $u(t), t \in J$ with property (2.2).

Theorem 2.2 Let $\left(\mathrm{H}_{1}\right)-\left(\mathrm{H}_{4}\right)$ hold. Assume that $0<f_{0} \leq f^{0}<+\infty, 0<I_{0}(k) \leq I^{0}(k)<+\infty$, $k=0,1, \ldots, p-1$ and the function $g$ satisfies (1.2). Then

(i) there exist $\lambda_{0}>0$ and $\mu_{0}>0$ such that, for any $\lambda>\lambda_{0}$ and $\mu>\mu_{0}$, problem (1.1) has a positive periodic solution $u(t)$ with

$$
\delta r \leq u(t) \leq R, \quad t \in J
$$

(ii) there exist $\bar{\lambda}_{0}>0$ and $\bar{\mu}_{0}>0$ such that, for any $0<\lambda<\bar{\lambda}_{0}$ and $0<\mu<\bar{\mu}_{0}$, problem (1.1) has a positive periodic solution $u(t), t \in J$ with property (2.3).

Remark 2.1 Some ideas of Theorems 2.1 and 2.2 are from Yan [28].

Theorem 2.3 Assume that $\left(\mathrm{H}_{1}\right)-\left(\mathrm{H}_{4}\right)$ hold and the function $g$ satisfies (1.2).

(i) If $f^{\infty}=0$ and $I^{\infty}(k)=0, k=0,1, \ldots, p-1$, then there exist $\lambda_{0}>0$ and $\mu_{0}>0$ such that, for any $\lambda>\lambda_{0}$ and $\mu>\mu_{0}$, problem (1.1) has a positive periodic solution $u(t)$, $t \in J$ with property (2.2).

(ii) If $f^{0}=0$ and $I^{0}(k)=0, k=0,1, \ldots, p-1$, then there exist $\lambda_{0}>0$ and $\mu_{0}>0$ such that, for any $\lambda>\lambda_{0}$ and $\mu>\mu_{0}$, problem (1.1) has a positive periodic solution $u(t)$ with property (2.3).

(iii) If $f^{0}=f^{\infty}=I^{\infty}(k)=I^{0}(k)=0, k=0,1, \ldots, p-1$, then there exist $\lambda_{0}>0$ and $\mu_{0}>0$ such that, for any $\lambda>\lambda_{0}$ and $\mu>\mu_{0}$, problem (1.1) has at least two positive periodic solutions $u_{1}(t)$ and $u_{2}(t)$ with

$$
\delta r \leq u_{1}(t) \leq r_{1}<\delta r_{2} \leq u_{2}(t) \leq R, \quad t \in J
$$


Theorem 2.4 Assume that $\left(\mathrm{H}_{1}\right)-\left(\mathrm{H}_{4}\right)$ hold and the function $g$ satisfies (1.2).

(i) If $f_{\infty}=+\infty$ and $I_{\infty}(k)=+\infty, k=0,1, \ldots, p-1$, then there exist $\bar{\lambda}_{0}>0$ and $\bar{\mu}_{0}>0$ such that, for any $0<\lambda<\bar{\lambda}_{0}$ and $0<\mu<\bar{\mu}_{0}$, problem (1.1) has a positive periodic solution $u(t), t \in J$ with property (2.2).

(ii) If $f_{0}=+\infty$ and $I_{0}(k)=+\infty, k=0,1, \ldots, p-1$, then there exist $\bar{\lambda}_{0}>0$ and $\bar{\mu}_{0}>0$ such that, for any $0<\lambda<\bar{\lambda}_{0}$ and $0<\mu<\bar{\mu}_{0}$, problem (1.1) has a positive periodic solution $u(t), t \in J$ with property (2.3).

(iii) If $f_{0}=f_{\infty}=I^{\infty}(k)=I_{0}(k)=+\infty, k=0,1, \ldots, p-1$, then there exist $\bar{\lambda}_{0}>0$ and $\bar{\mu}_{0}>0$ such that, for any $0<\lambda<\bar{\lambda}_{0}$ and $0<\mu<\bar{\mu}_{0}$, problem (1.1) has at least two positive periodic solutions $u_{1}(t)$ and $u_{2}(t)$ with

$$
\delta r \leq u_{1}(t) \leq r_{1}<\delta r_{2} \leq u_{2}(t) \leq \frac{1}{\delta} R, \quad t \in J
$$

Remark 2.2 Some ideas of the proof of Theorem 2.3 and Theorem 2.4 are from Wang [10].

\subsection{Preliminaries}

In our main results, we will make use of the following definitions and lemmas.

Definition 2.1 A function $u: \mathbf{R} \rightarrow(0, \infty)$ is called a positive solution of (1.1) if it satisfies:

(i) $u(t)$ is absolutely continuous on each $\left(t_{k}, t_{k+1}\right)$;

(ii) for each $k \in \mathbf{Z}, u\left(t_{k}^{+}\right)$and $u\left(t_{k}^{-}\right)$exist and $u\left(t_{k}^{-}\right)=u\left(t_{k}\right)$;

(iii) $u(t)$ satisfies (1.1).

Definition 2.2 A solution $u(t)$ of (1.1) on $\mathbf{R}$ is called $\omega$-periodic if $u(t+\omega)=u(t)$.

Let $E$ be the Banach space

$\left\{u \mid u: \mathbf{R} \rightarrow \mathbf{R}\right.$ is continuous at $t \neq t_{k}, u\left(t_{k}^{-}\right)=u\left(t_{k}\right)$ and

$u\left(t_{k}^{+}\right)$exists, $k \in \mathbf{Z}$ and $\left.u(t+\omega)=u(t)\right\}$

with $\|u\|=\sup _{0 \leq t \leq \omega}|u(t)|$. We denote

$$
\Omega_{r}:=\{u \in E:\|u\|<r\}, \quad \partial \Omega=\{u \in E:\|u\|=r\}
$$

for all $r>0$ in the sequel.

Lemma 2.1 (See [34]) Assume that $\left(\mathrm{H}_{1}\right)-\left(\mathrm{H}_{4}\right)$ hold and the function $g$ satisfies (1.2). Then $u \in E$ is a solution of problem (1.1) if and only if $u$ is a solution of the integral equation

$$
\begin{aligned}
u(t)= & \lambda \int_{t}^{t+\omega} G(t, s) b(s) f\left(s, u\left(h_{2}(s)\right), \int_{-\zeta}^{0} e(s) u(s+v) d v\right) d s \\
& +\mu \sum_{t \leq t_{k}<t+\omega} G\left(t, t_{k}\right) I_{k}\left(t_{k}, u\left(t_{k}\right)\right),
\end{aligned}
$$

where

$$
G(t, s)=\frac{e^{\int_{t}^{s} a(v) g\left(u\left(h_{1}(v)\right)\right) d v}}{e^{\int_{0}^{\omega} a(v) g\left(u\left(h_{1}(v)\right)\right) d v}-1}, \quad s \in[t, t+\omega]
$$


Further, it follows from (2.7) and (1.2) that

$$
\frac{1}{\sigma^{L}-1}=\alpha \leq G(t, s) \leq \beta=\frac{\sigma^{L}}{\sigma^{l}-1}, \quad s \in[t, t+\omega]
$$

where $\sigma=e^{\int_{0}^{\omega} a(t) d t}$.

Define a cone $K$ in $E$ by

$$
K=\{u \in E: u(t) \geq \delta\|u\|, t \in J\}
$$

where

$$
0<\delta=\frac{\sigma^{l}-1}{\sigma^{L}\left(\sigma^{L}-1\right)}<1
$$

It is easy to see $K$ is a closed non-empty subset of $E$.

Define $T_{\lambda}^{\mu}: K \rightarrow E$ by

$$
\begin{aligned}
\left(T_{\lambda}^{\mu} u\right)(t)= & \lambda \int_{t}^{t+\omega} G(t, s) b(s) f\left(s, u\left(h_{2}(s)\right), \int_{-\zeta}^{0} e(s) u(s+v) d v\right) d s \\
& +\mu \sum_{t \leq t_{k}<t+\omega} G\left(t, t_{k}\right) I_{k}\left(t_{k}, u\left(t_{k}\right)\right) .
\end{aligned}
$$

From (2.10) and Lemma 2.1, it is easy to obtain the following result.

Lemma 2.2 (See [16]) Assume that $\left(\mathrm{H}_{1}\right)-\left(\mathrm{H}_{4}\right)$ hold. Problem (1.1) is equivalent to the fixed point problem of $T_{\lambda}^{\mu}$ in $K$.

Lemma 2.3 (See Lemmas 2.1 and 2.2 in [28]) Assume that $\left(\mathrm{H}_{1}\right)-\left(\mathrm{H}_{4}\right)$ hold and the function $g$ satisfies (1.2). Then $T_{\lambda}^{\mu}: K \rightarrow K$ is completely continuous.

The following well-known result of the fixed point is crucial in our arguments.

Lemma 2.4 (See [45]) Let $P$ be a cone in a real Banach space E. Assume $\Omega_{1}, \Omega_{2}$ are bounded open sets in $E$ with $0 \in \Omega_{1}, \bar{\Omega}_{1} \subset \Omega_{2}$. If

$$
A: P \cap\left(\bar{\Omega}_{2} \backslash \Omega_{1}\right) \rightarrow P
$$

is completely continuous such that either

(a) $\|A x\| \leq\|x\|, \forall x \in P \cap \partial \Omega_{1}$ and $\|A x\| \geq\|x\|, \forall x \in P \cap \partial \Omega_{2}$, or

(b) $\|A x\| \geq\|x\|, \forall x \in P \cap \partial \Omega_{1}$ and $\|A x\| \leq\|x\|, \forall x \in P \cap \partial \Omega_{2}$, then $A$ has at least one fixed point in $P \cap\left(\bar{\Omega}_{2} \backslash \Omega_{1}\right)$.

\subsection{Proofs of the main results}

For convenience we write

$$
\gamma=\int_{0}^{\omega} b(s) d s
$$


Proof of Theorem 2.1 Part (i). Noticing that $f(t, x, y)>0, I_{k}(t, x)>0(k=0,1, \ldots, p-1)$ for all $t, x>0$, and $y>0$, we can define, for $r>0$,

$$
m_{r}=\min _{t \in J, \delta r \leq x, y \leq r}\{f(t, x, y)\}>0, \quad m^{*}=\min \left\{m_{k}, k=0,1, \ldots, p-1\right\}>0,
$$

where

$$
m_{k}=\min _{t \in J, \delta r \leq x \leq r}\left\{I_{k}(t, x)\right\}>0, \quad k=0,1, \ldots, p-1 .
$$

Since $\int_{-\zeta}^{0} e(s) d s=1$, it follows from $\delta r \leq x \leq r$ that

$$
\delta r \leq \int_{-\zeta}^{0} e(s) x(s+v) d v \leq r
$$

Let

$$
\lambda_{0} \geq\left(2 \alpha m_{r} \gamma\right)^{-1} r, \quad \mu_{0} \geq\left(2 \alpha m^{*} p\right)^{-1} r .
$$

Then for any $u \in K \cap \partial \Omega_{r}$ and $\lambda>\lambda_{0}, \mu>\mu_{0}$, we have

$$
\begin{aligned}
\left(T_{\lambda}^{\mu} u\right)(t)= & \lambda \int_{t}^{t+\omega} G(t, s) b(s) f\left(s, u\left(h_{2}(s)\right), \int_{-\zeta}^{0} e(s) u(s+v) d v\right) d s \\
& +\mu \sum_{t \leq t_{k}<t+\omega} G\left(t, t_{k}\right) I_{k}\left(t_{k}, u\left(t_{k}\right)\right) \\
\geq & \alpha\left[\lambda \int_{0}^{\omega} b(s) f\left(s, u\left(h_{2}(s)\right), \int_{-\zeta}^{0} e(s) u(s+v) d v\right) d s+\mu \sum_{0 \leq t_{k}<\omega} I_{k}\left(t_{k}, u\left(t_{k}\right)\right)\right] \\
\geq & \alpha\left[\lambda \int_{0}^{\omega} b(s) f\left(s, u\left(h_{2}(s)\right), \int_{-\zeta}^{0} e(s) u(s+v) d v\right) d s+\mu \sum_{k=0} I_{k}\left(t_{k}, u\left(t_{k}\right)\right)\right] \\
\geq & \alpha\left[\lambda m_{r} \int_{0}^{\omega} b(s) d s+\mu m^{*} p\right] \\
> & \alpha\left[\lambda_{0} m_{r} \gamma+\mu_{0} m^{*} p\right] \\
\geq & \frac{1}{2} r+\frac{1}{2} r=r
\end{aligned}
$$

which implies that

$$
\left\|T_{\lambda}^{\mu} u\right\|>\|u\|, \quad \forall u \in K \cap \partial \Omega_{r}, \lambda>\lambda_{0} \text { and } \mu>\mu_{0} .
$$

If $0<f^{\infty}<+\infty, 0<I^{\infty}(k)<+\infty, k=0,1, \ldots, p-1$, then there exist $l_{1}>0, l_{2}>0$, and $R>r>0$ such that

$$
f(t, x, y)<l_{1} x, \quad I_{k}(t, x)<l_{2} x \quad(\forall t \in J, x \geq R, y \geq R, k=0,1, \ldots, p-1),
$$

where $l_{1}$ satisfies

$$
2 l_{1} \beta \lambda \gamma \leq 1
$$


$l_{2}$ satisfies

$$
2 l_{2} \beta \mu p \leq 1 .
$$

Let $\eta=\frac{R}{\delta}$. Thus, when $u \in K \cap \partial \Omega_{\eta}$ we have

$$
u(t) \geq \delta\|u\|=\delta \eta=R, \quad t \in J .
$$

Since $\int_{-\zeta}^{0} e(s) d s=1$, it follows from $x \geq R$ that

$$
\int_{-\zeta}^{0} e(s) x(s+v) d v \geq R
$$

Therefore, it follows from (2.10) that

$$
\begin{aligned}
\left(T_{\lambda}^{\mu} u\right)(t)= & \lambda \int_{t}^{t+\omega} G(t, s) b(s) f\left(s, u\left(h_{2}(s)\right), \int_{-\zeta}^{0} e(s) u(s+v) d v\right) d s \\
& +\mu \sum_{t \leq t_{k}<t+\omega} G\left(t, t_{k}\right) I_{k}\left(t_{k}, u\left(t_{k}\right)\right) \\
\leq & \beta\left[\lambda \int_{0}^{\omega} b(s) f\left(s, u\left(h_{2}(s)\right), \int_{-\zeta}^{0} e(s) u(s+v) d v\right) d s+\mu \sum_{0 \leq t_{k}<\omega} I_{k}\left(t_{k}, u\left(t_{k}\right)\right)\right] \\
\leq & \beta\left[\lambda \int_{0}^{\omega} b(s) f\left(s, u\left(h_{2}(s)\right), \int_{-\zeta}^{0} e(s) u(s+v) d v\right) d s+\mu \sum_{k=0}^{p-1} I_{k}\left(t_{k}, u\left(t_{k}\right)\right)\right] \\
\leq & \beta\left[\lambda \int_{0}^{\omega} b(s) l_{1}\|u\| d s+\mu \sum_{k=0}^{p-1} l_{2}\|u\|\right] \\
\leq & \beta\left[\lambda l_{1} \int_{0}^{\omega} b(s) d s\|u\|+\mu l_{2} p\|u\|\right] \\
\leq & \beta\left[\lambda l_{1} \gamma\|u\|+\mu l_{2} p\|u\|\right] \\
\leq & \frac{1}{2}\|u\|+\frac{1}{2}\|u\| \\
= & \|u\| .
\end{aligned}
$$

This yields

$$
\left\|T_{\lambda}^{\mu} u\right\| \leq\|u\|, \quad \forall u \in K \cap \partial \Omega_{\eta} .
$$

Applying (b) of Lemma 2.4 to (2.11) and (2.14) shows that $T_{\lambda}^{\mu}$ has a fixed point $u \in K \cap$ $\left(\bar{\Omega}_{\eta} \backslash \Omega_{r}\right)$ with $r \leq\|u\| \leq \eta=\frac{1}{\delta} R$. Hence, since for $u \in K$ we have $u(t) \geq \delta\|u\|, t \in J$, it follows that (2.2) holds. This gives the proof of part (i).

Part (ii). Noticing that $f(t, x, y)>0, I_{k}(t, x)>0(k=0,1, \ldots, p-1)$ for all $t, x>0$, and $y>0$, we can define, for $r>0$,

$$
M_{r}=\max _{t \in J, 0 \leq x, y \leq r}\{f(t, x, y)\}>0, \quad M^{*}=\max \left\{M_{k}, k=0,1, \ldots, p-1\right\}>0,
$$


where

$$
M_{k}=\max _{t \in J, 0 \leq x \leq r}\left\{I_{k}(t, x)\right\}, \quad k=0,1, \ldots, p-1 .
$$

Since $\int_{-\zeta}^{0} e(s) d s=1$, it follows from $0 \leq x \leq r$ that

$$
0 \leq \int_{-\zeta}^{0} e(s) x(s+v) d v \leq r
$$

Let

$$
\bar{\lambda}_{0} \leq \frac{1}{2 \beta M_{r} \gamma} r, \quad \bar{\mu}_{0} \leq \frac{1}{2 \beta p M^{*}} r .
$$

Then, for $u \in K \cap \partial \Omega_{r}$ and $\lambda<\bar{\lambda}_{0}, \mu<\bar{\mu}_{0}$, we have

$$
\begin{aligned}
\left(T_{\lambda}^{\mu} u\right)(t)= & \lambda \int_{t}^{t+\omega} G(t, s) b(s) f\left(s, u\left(h_{2}(s)\right), \int_{-\zeta}^{0} e(s) u(s+v) d v\right) d s \\
& +\mu \sum_{t \leq t_{k}<t+\omega} G\left(t, t_{k}\right) I_{k}\left(t_{k}, u\left(t_{k}\right)\right) \\
\leq & \beta\left[\lambda \int_{0}^{\omega} b(s) f\left(s, u\left(h_{2}(s)\right), \int_{-\zeta}^{0} e(s) u(s+v) d v\right) d s+\mu \sum_{0 \leq t_{k}<\omega} I_{k}\left(t_{k}, u\left(t_{k}\right)\right)\right] \\
\leq & \beta\left[\lambda \int_{0}^{\omega-1} b(s) f\left(s, u\left(h_{2}(s)\right), \int_{-\zeta}^{0} e(s) u(s+v) d v\right) d s+\mu \sum_{k=0}^{p} I_{k}\left(t_{k}, u\left(t_{k}\right)\right)\right] \\
\leq & \beta\left[\lambda \int_{0}^{\omega} b(s) M_{r} d s+\mu \sum_{k=0}^{p-1} M^{*}\right] \\
\leq & \beta\left[\lambda M_{r} \int_{0}^{\omega} b(s) d s+\mu p M^{*}\right] \\
\leq & \beta\left[\lambda M_{r} \gamma+\mu M^{*} p\right] \\
< & \beta\left[\bar{\lambda}_{0} M_{r} \gamma+\bar{\mu}_{0} M^{*} p\right] \\
\leq & \frac{1}{2} r+\frac{1}{2} r \\
= & r .
\end{aligned}
$$

This implies

$$
\left\|T_{\lambda}^{\mu} u\right\|<\|u\|, \quad \forall u \in K \cap \partial \Omega_{r} .
$$

If $0<f_{\infty}<+\infty, 0<I_{\infty}(k)<+\infty, k=0,1, \ldots, p-1$, then there exist $l_{3}>0, l_{4}>0$, and $R>r>0$ such that

$$
f(t, x, y)>l_{3} x, \quad I_{k}(t, x)>l_{4} x \quad(\forall t \in J, x \geq R, y \geq R, k=0,1, \ldots, p-1),
$$

where $l_{3}$ satisfies

$$
2 \alpha \delta \lambda l_{3} \gamma \geq 1
$$


$l_{4}$ satisfies

$$
2 \alpha \delta \mu l_{4} p \geq 1
$$

Let $\bar{\eta}=\frac{R}{\delta}$. Thus, when $u \in K \cap \partial \Omega_{\bar{\eta}}$ we have

$$
u(t) \geq \delta\|u\|=\delta \bar{\eta}=R, \quad t \in J .
$$

Since $\int_{-\zeta}^{0} e(s) d s=1$, it follows from $x \geq R$ that

$$
\int_{-\zeta}^{0} e(s) x(s+v) d v \geq R
$$

Therefore, for any $u \in K \cap \partial \Omega_{\bar{\eta}}$, we have

$$
\begin{aligned}
\left(T_{\lambda}^{\mu} u\right)(t)= & \lambda \int_{t}^{t+\omega} G(t, s) b(s) f\left(s, u\left(h_{2}(s)\right), \int_{-\zeta}^{0} e(s) u(s+v) d v\right) d s \\
& +\mu \sum_{t \leq t_{k}<t+\omega} G\left(t, t_{k}\right) I_{k}\left(t_{k}, u\left(t_{k}\right)\right) \\
\geq & \alpha\left[\lambda \int_{0}^{\omega} b(s) f\left(s, u\left(h_{2}(s)\right), \int_{-\zeta}^{0} e(s) u(s+v) d v\right) d s+\mu \sum_{0 \leq t_{k}<\omega} I_{k}\left(t_{k}, u\left(t_{k}\right)\right)\right] \\
\geq & \alpha\left[\lambda \int_{0}^{\omega} b(s) f\left(s, u\left(h_{2}(s)\right), \int_{-\zeta}^{0} e(s) u(s+v) d v\right) d s+\mu \sum_{k=0}^{p-1} I_{k}\left(t_{k}, u\left(t_{k}\right)\right)\right] \\
\geq & \alpha\left[\lambda \int_{0}^{\omega} b(s) l_{3} \delta\|u\| d s+\mu l_{4} \delta\|u\| p\right] \\
\geq & \alpha \delta\left(\lambda l_{3} \gamma\|u\|+\mu l_{4} p\|u\|\right) \\
\geq & \frac{1}{2}\|u\|+\frac{1}{2}\|u\| \\
= & \|u\|,
\end{aligned}
$$

which implies that

$$
\left\|T_{\lambda}^{\mu} u\right\| \geq\|u\|, \quad \forall u \in K \cap \partial \Omega_{\bar{\eta}} .
$$

Applying (a) of Lemma 2.4 to (2.15) and (2.18) shows that $T_{\lambda}^{\mu}$ has a fixed point $u \in K \cap$ $\left(\bar{\Omega}_{\bar{\eta}} \backslash \Omega_{\bar{r}}\right)$ with $r \leq\|u\| \leq \bar{\eta}=\frac{1}{\delta} R$. Hence, since for $u \in K$ we have $u(t) \geq \delta\|u\|, t \in J$, it follows that (2.3) holds. This finishes the proof of part (ii).

Proof of Theorem 2.2 Part (i). Noticing that $f(t, x, y)>0, I_{k}(t, x)>0(k=0,1, \ldots, p-1)$ for all $t, x>0$, and $y>0$, we can define, for $R>0$,

$$
m_{R}=\min _{t \in J, \delta R \leq x, y \leq R}\{f(t, x, y)\}>0, \quad m^{*}=\min \left\{m_{k}, k=0,1, \ldots, p-1\right\}>0,
$$

where

$$
m_{k}=\min _{t \in J, \delta R \leq x \leq R}\left\{I_{k}(t, x)\right\}, \quad k=0,1, \ldots, p-1 .
$$


Let

$$
\lambda_{0} \geq\left(2 \alpha m_{R} \gamma\right)^{-1} R, \quad \mu_{0} \geq\left(2 \alpha m^{*} p\right)^{-1} R .
$$

Then, for any $u \in K \cap \partial \Omega_{R}$ and $\lambda>\lambda_{0}, \mu>\mu_{0}$, we have

$$
\begin{aligned}
\left(T_{\lambda}^{\mu} u\right)(t)= & \lambda \int_{t}^{t+\omega} G(t, s) b(s) f\left(s, u\left(h_{2}(s)\right), \int_{-\zeta}^{0} e(s) u(s+v) d v\right) d s \\
& +\mu \sum_{t \leq t_{k}<t+\omega} G\left(t, t_{k}\right) I_{k}\left(t_{k}, u\left(t_{k}\right)\right) \\
\geq & \alpha\left[\lambda \int_{0}^{\omega} b(s) f\left(s, u\left(h_{2}(s)\right), \int_{-\zeta}^{0} e(s) u(s+v) d v\right) d s+\mu \sum_{0 \leq t_{k}<\omega} I_{k}\left(t_{k}, u\left(t_{k}\right)\right)\right] \\
\geq & \alpha\left[\lambda \int_{0}^{\omega} b(s) f\left(s, u\left(h_{2}(s)\right), \int_{-\zeta}^{0} e(s) u(s+v) d v\right) d s+\mu \sum_{k=0}^{p-1} I_{k}\left(t_{k}, u\left(t_{k}\right)\right)\right] \\
\geq & \alpha\left[\lambda m_{R} \int_{0}^{\omega} b(s) d s+\mu m^{*} p\right] \\
> & \alpha\left[\lambda_{0} m_{R} \gamma+\mu_{0} m^{*} p\right] \\
\geq & \frac{1}{2} R+\frac{1}{2} R=R,
\end{aligned}
$$

which implies that

$$
\left\|T_{\lambda}^{\mu} u\right\|>\|u\|, \quad \forall u \in K \cap \partial \Omega_{R}, \lambda>\lambda_{0} \text { and } \mu>\mu_{0}
$$

If $0<f^{0}<+\infty, 0<I^{0}(k)<+\infty, k=0,1, \ldots, p-1$, then there exist $l_{1}>0, l_{2}>0$, and $0<$ $r<R$ such that

$$
f(t, x, y)<l_{1} x, \quad I_{k}(t, x)<l_{2} x \quad(\forall t \in J, 0 \leq x, y \leq r, k=0,1, \ldots, p-1),
$$

where $l_{1}$ and $l_{2}$ satisfy (2.12) and (2.13), respectively.

Therefore, for $u \in K \cap \partial \Omega_{r}$, we have

$$
\begin{aligned}
\left(T_{\lambda}^{\mu} u\right)(t)= & \lambda \int_{t}^{t+\omega} G(t, s) b(s) f\left(s, u\left(h_{2}(s)\right), \int_{-\zeta}^{0} e(s) u(s+v) d v\right) d s \\
& +\mu \sum_{t \leq t_{k}<t+\omega} G\left(t, t_{k}\right) I_{k}\left(t_{k}, u\left(t_{k}\right)\right) \\
\leq & \beta\left[\lambda \int_{0}^{\omega} b(s) f\left(s, u\left(h_{2}(s)\right), \int_{-\zeta}^{0} e(s) u(s+v) d v\right) d s+\mu \sum_{0 \leq t_{k}<\omega} I_{k}\left(t_{k}, u\left(t_{k}\right)\right)\right] \\
\leq & \beta\left[\lambda \int_{0}^{\omega} b(s) f\left(s, u\left(h_{2}(s)\right), \int_{-\zeta}^{0} e(s) u(s+v) d v\right) d s+\mu \sum_{k=0}^{p-1} I_{k}\left(t_{k}, u\left(t_{k}\right)\right)\right] \\
\leq & \beta\left[\lambda \int_{0}^{\omega} b(s) l_{1}\|u\| d s+\mu \sum_{k=0}^{p-1} l_{2}\|u\|\right] \\
\leq & \beta\left[\lambda l_{1} \int_{0}^{\omega} b(s) d s\|u\|+\mu l_{2} p\|u\|\right]
\end{aligned}
$$




$$
\begin{aligned}
& \leq \beta\left[\lambda l_{1} \gamma\|u\|+\mu l_{2} p\|v\|\right] \\
& \leq \frac{1}{2}\|u\|+\frac{1}{2}\|u\| \\
& =\|u\| .
\end{aligned}
$$

This yields

$$
\left\|T_{\lambda}^{\mu} u\right\| \leq\|u\|, \quad \forall u \in K \cap \partial \Omega_{r}
$$

Applying (a) of Lemma 2.4 to (2.19) and (2.20) shows that $T_{\lambda}^{\mu}$ has a fixed point $u \in$ $K \cap\left(\bar{\Omega}_{R} \backslash \Omega_{r}\right)$ with $r \leq\|u\| \leq R$. Hence, since for $u \in K$ we have $u(t) \geq \delta\|u\|, t \in J$, it follows that (2.3) holds. This gives the proof of part (i).

Part (ii). Noticing that $f(t, x, y)>0, I_{k}(t, x)>0(k=0,1, \ldots, p-1)$ for all $t, x>0$, and $y>0$, we can define, for $R>0$,

$$
M_{R}=\max _{t \in J, 0 \leq x, y \leq R}\{f(t, x, y)\}>0, \quad M^{*}=\max \left\{M_{k}, k=0,1, \ldots, p-1\right\}>0,
$$

where

$$
M_{k}=\max _{t \in J, 0 \leq x \leq R}\left\{I_{k}(t, x)\right\}, \quad k=0,1, \ldots, p-1 .
$$

Let

$$
\bar{\lambda}_{0} \leq \frac{1}{2 \beta M_{R} \gamma} R, \quad \bar{\mu}_{0} \leq \frac{1}{2 \beta p M^{*}} R .
$$

Then, for $u \in K \cap \partial \Omega_{R}$ and $\lambda<\bar{\lambda}_{0}, \mu<\bar{\mu}_{0}$, we have

$$
\begin{aligned}
\left(T_{\lambda}^{\mu} u\right)(t)= & \lambda \int_{t}^{t+\omega} G(t, s) b(s) f\left(s, u\left(h_{2}(s)\right), \int_{-\zeta}^{0} e(s) u(s+v) d v\right) d s \\
& +\mu \sum_{t \leq t_{k}<t+\omega} G\left(t, t_{k}\right) I_{k}\left(t_{k}, u\left(t_{k}\right)\right) \\
\leq & \beta\left[\lambda \int_{0}^{\omega} b(s) f\left(s, u\left(h_{2}(s)\right), \int_{-\zeta}^{0} e(s) u(s+v) d v\right) d s+\mu \sum_{0 \leq t_{k}<\omega} I_{k}\left(t_{k}, u\left(t_{k}\right)\right)\right] \\
\leq & \beta\left[\lambda \int_{0}^{\omega} b(s) f\left(s, u\left(h_{2}(s)\right), \int_{-\zeta}^{0} e(s) u(s+v) d v\right) d s+\mu \sum_{k=0}^{p-1} I_{k}\left(t_{k}, u\left(t_{k}\right)\right)\right] \\
\leq & \beta\left[\lambda \int_{0}^{\omega} b(s) M_{R} d s+\mu \sum_{k=0}^{p-1} M^{*}\right] \\
\leq & \beta\left[\lambda M_{R} \int_{0}^{\omega} b(s) d s+\mu p M^{*}\right] \\
\leq & \beta\left[\lambda M_{R} \gamma+\mu M^{*} p\right] \\
< & \beta\left[\bar{\lambda}_{0} M_{R} \gamma+\bar{\mu}_{0} M^{*} p\right] \\
\leq & \frac{1}{2} R+\frac{1}{2} R \\
= & R .
\end{aligned}
$$


This implies

$$
\left\|T_{\lambda}^{\mu} u\right\|<\|u\|, \quad \forall u \in K \cap \partial \Omega_{R}
$$

If $0<f_{0}<+\infty, 0<I_{0}(k)<+\infty, k=0,1, \ldots, p-1$, then there exist $l_{3}>0, l_{4}>0$, and $0<r<$ $R$ such that

$$
f(t, x, y)>l_{3} x, \quad I_{k}(t, x)>l_{4} x \quad(\forall t \in J, 0 \leq x, y \leq r, k=0,1, \ldots, p-1),
$$

where $l_{3}$ and $l_{4}$ satisfy (2.16) and (2.17), respectively.

Therefore, for any $u \in K \cap \partial \Omega_{r}$, we have

$$
\begin{aligned}
\left(T_{\lambda}^{\mu} u\right)(t)= & \lambda \int_{t}^{t+\omega} G(t, s) b(s) f\left(s, u\left(h_{2}(s)\right), \int_{-\zeta}^{0} e(s) u(s+v) d v\right) d s \\
& +\mu \sum_{t \leq t_{k}<t+\omega} G\left(t, t_{k}\right) I_{k}\left(t_{k}, u\left(t_{k}\right)\right) \\
\geq & \alpha\left[\lambda \int_{0}^{\omega} b(s) f\left(s, u\left(h_{2}(s)\right), \int_{-\zeta}^{0} e(s) u(s+v) d v\right) d s+\mu \sum_{0 \leq t_{k}<\omega} I_{k}\left(t_{k}, u\left(t_{k}\right)\right)\right] \\
\geq & \alpha\left[\lambda \int_{0}^{\omega} b(s) f\left(s, u\left(h_{2}(s)\right), \int_{-\zeta}^{0} e(s) u(s+v) d v\right) d s+\mu \sum_{k=0} I_{k}\left(t_{k}, u\left(t_{k}\right)\right)\right] \\
\geq & \alpha\left[\lambda \int_{0}^{\omega} b(s) l_{3}\|u\| d s+\mu l_{4}\|u\| p\right] \\
\geq & \alpha\left[\lambda l_{3}\|u\| \gamma+\mu l_{4}\|u\| p\right] \\
\geq & \frac{1}{2}\|u\|+\frac{1}{2}\|u\| \\
= & \|u\|,
\end{aligned}
$$

which implies that

$$
\left\|T_{\lambda}^{\mu} u\right\| \geq\|u\|, \quad \forall u \in K \cap \partial \Omega_{r}
$$

Applying (a) of Lemma 2.4 to (2.21) and (2.22) shows that $T_{\lambda}^{\mu}$ has a fixed point $u \in$ $K \cap\left(\bar{\Omega}_{R} \backslash \Omega_{r}\right)$ with $r \leq\|u\| \leq R$. Hence, since for $u \in K$ we have $u(t) \geq \delta\|u\|, t \in J$, it follows that (2.3) holds. This finishes the proof of part (ii).

Proof of Theorem 2.3 Similar to the proof of Theorem 2.1(i) and Theorem 2.2(i), respectively, one can show that Theorem 2.3(i) and (ii) hold.

Consider part (iii). Choose two numbers $r_{1}$ and $r_{2}$ satisfying (2.1). By Theorem 2.1(i) and Theorem 2.2(i), there exist $\lambda_{0}>0$ and $\mu_{0}>0$ such that

$$
\left\|T_{\lambda}^{\mu} u\right\|>\|u\|, \quad \forall u \in K \cap \partial \Omega_{r_{i}}, i=1,2 .
$$

Since $f^{0}=f^{\infty}=I^{\infty}(k)=I^{0}(k)=0, k=0,1, \ldots, p-1$, from the proof of Theorem 2.1(i), Theorem 2.2(i), and (2.1), it follows that

$$
\left\|T_{\lambda}^{\mu} u\right\| \leq\|u\|, \quad \forall u \in K \cap \partial \Omega_{r}
$$


and

$$
\left\|T_{\lambda}^{\mu} u\right\| \leq\|u\|, \quad \forall u \in K \cap \partial \Omega_{R}
$$

Applying Lemma 2.4 to (2.23)-(2.25) shows that $T_{\lambda}^{\mu}$ has two fixed points $u_{1}$ and $u_{2}$ such that $u_{1} \in K \cap\left(\bar{\Omega}_{r_{1}} \backslash \Omega_{r}\right)$ and $u_{2} \in K \cap\left(\bar{\Omega}_{R} \backslash \Omega_{r_{2}}\right)$. These are the desired distinct positive periodic solutions of problem (1.1) for $\lambda_{0}>0$ and $\mu_{0}>0$ satisfying (2.4). Then the result of part (iii) follows.

Proof of Theorem 2.4 Similar to the proof of those Theorem 2.1(ii) and Theorem 2.2(ii), respectively, one can show that Theorem 2.4(i) and (ii) hold.

Now consider part (iii). Choose two numbers $r_{1}$ and $r_{2}$ satisfying (2.1). By Theorem 2.1(ii) and Theorem 2.2(ii), there exist $\bar{\lambda}_{0}>0$ and $\bar{\mu}_{0}>0$ such that

$$
\left\|T_{\lambda}^{\mu} u\right\|<\|u\|, \quad \forall 0<\lambda<\bar{\lambda}_{0}, 0<\mu<\bar{\mu}_{0}, u \in K \cap \partial \Omega_{r_{i}}, i=1,2 .
$$

Since $f_{0}=f_{\infty}=I_{\infty}(k)=I_{0}(k)=+\infty, k=0,1, \ldots, p-1$, from the proof of Theorem 2.1(ii) and Theorem 2.2(ii) and (2.1), it follows that

$$
\left\|T_{\lambda}^{\mu} u\right\| \geq\|u\|, \quad \forall u \in K \cap \partial \Omega_{r}
$$

and

$$
\left\|T_{\lambda}^{\mu} u\right\| \geq\|u\|, \quad \forall u \in K \cap \partial \Omega_{R}
$$

Applying Lemma 2.4 to (2.26)-(2.28) shows that $T_{\lambda}^{\mu}$ has two fixed points $u_{1}$ and $u_{2}$ such that $u_{1} \in K \cap\left(\bar{\Omega}_{r_{1}} \backslash \Omega_{r}\right)$ and $u_{2} \in K \cap\left(\bar{\Omega}_{R} \backslash \Omega_{r_{2}}\right)$. These are the desired distinct positive periodic solutions of problem (1.1) for $0<\lambda<\bar{\lambda}_{0}$ and $0<\mu<\bar{\mu}_{0}$ satisfying (2.5). Then proof of part (iii) is complete.

\section{The parameter dependence of positive periodic solution under (1.2)}

In this section, we consider the parameter dependence of positive periodic solution in the case $\lambda=\mu$. For convenience, let

$$
\mathbf{M}=\max _{t \in J, 0 \leq x, y \leq d} f(t, x, y), \quad \mathbf{M}^{*}=\max \left\{\mathbf{M}_{k}, k=0,1, \ldots, p-1\right\},
$$

where

$$
\mathbf{M}_{k}=\max _{t \in J, 0 \leq x \leq d} I_{k}(t, x), \quad k=0,1, \ldots, p-1,
$$

and $d$ is a positive number.

$\left(\mathrm{H}_{3}\right)^{\prime}$ Let $f \in C\left(\mathbf{R} \times \mathbf{R}_{+} \times \mathbf{R}_{+}, \mathbf{R}_{+}\right)$be $\omega$-periodic functions in $t$.

Theorem 3.1 Assume that $\left(\mathrm{H}_{1}\right),\left(\mathrm{H}_{2}\right),\left(\mathrm{H}_{3}\right)^{\prime}$, and $\left(\mathrm{H}_{4}\right)$ hold and the function $g$ satisfies (1.2). Then the following two conclusions hold. 
$\left(\mathrm{H}_{5}\right)$ If $f^{0}=0, I^{0}(k)=0$, and $f_{\infty}=\infty, I_{\infty}(k)=\infty, k=0,1, \ldots, p-1$, then for every $\lambda>0$ problem (1.1) has a positive periodic solution $u_{\lambda}(t)$ satisfying $\lim _{\lambda \rightarrow 0^{+}}\left\|u_{\lambda}\right\|=\infty$.

$\left(\mathrm{H}_{6}\right)$ If $f_{0}=\infty, I_{0}(k)=\infty$, and $f^{\infty}=0, I^{\infty}(k)=0, k=0,1, \ldots, p-1$, then for every $\lambda>0$ problem (1.1) has a positive periodic solution $u_{\lambda}(t)$ satisfying $\lim _{\lambda \rightarrow 0^{+}}\left\|u_{\lambda}\right\|=0$.

Remark 3.1 Assume that $f_{\infty}=\infty$ or $I_{\infty}(k)=\infty, k=0,1, \ldots, p-1$ in $\left(\mathrm{H}_{5}\right)$ or $f_{0}=\infty$ or $I_{0}(k)=\infty, k=0,1, \ldots, p-1$ in $\left(\mathrm{H}_{6}\right)$. Then the conclusions of Theorem 3.1 also hold.

Remark 3.2 Theorem 3.1 improves and develops Theorem 2.3.7 in [45] essentially.

Remark 3.3 Theorem 3.1 only considers the special case $\mu=\lambda$. However, we do not give any information on the general case $\mu \neq \lambda$.

Proof of Theorem 3.1 We need only to prove this theorem under condition $\left(\mathrm{H}_{5}\right)$ since the proof is similar when $\left(\mathrm{H}_{6}\right)$ holds.

For given $\lambda>0$, similar to the proof of (2.18) and (2.20), it follows from $\left(\mathrm{H}_{5}\right)$ that there exists $0<r<R$ such that

$$
\left\|T_{\lambda}^{\mu} u\right\| \leq\|u\|, \quad u \in K \cap \partial \Omega_{r}
$$

and

$$
\left\|T_{\lambda}^{\mu} T u\right\| \geq\|u\|, \quad u \in K \cap \partial \Omega_{R}
$$

Hence, condition (a) of Lemma 2.4 is satisfied for the operator $T_{\lambda}^{\mu}$, which implies that $T_{\lambda}^{\mu}$ has a fixed point $u_{\lambda}$ in $\bar{\Omega}_{R} \backslash \Omega_{r}$.

It remains to prove $\left\|u_{\lambda}\right\|=+\infty$ as $\lambda \rightarrow 0^{+}$. In fact, if not, there exist a number $d>0$ and a sequence $\lambda_{n} \rightarrow 0^{+}$such that

$$
\left\|u_{\lambda_{n}}\right\| \leq d \quad(n=1,2,3, \ldots)
$$

Furthermore, the sequence $\left\{\left\|u_{\lambda_{n}}\right\|\right\}$ contains a subsequence that converges to a number $\eta$ $(0 \leq \eta \leq d)$. For simplicity, suppose that $\left\{\left\|u_{\lambda_{n}}\right\|\right\}$ itself converges to $\eta$.

If $\eta>0$, then $\left\|u_{\lambda_{n}}\right\|>\frac{\eta}{2}$ for sufficiently large $n(n>\mathbf{N})$, and therefore

$$
\begin{aligned}
\frac{1}{\lambda_{n}} & =\frac{\left\|\int_{t}^{t+\omega} G(t, s) b(s) f\left(s, u\left(h_{2}(s)\right), \int_{-\zeta}^{0} e(s) u(s+v) d v\right) d s+\sum_{t \leq t_{k} \leq t+\omega} G\left(t, t_{k}\right) I_{k}\left(t_{k}, u_{\lambda_{n}}\left(t_{k}\right)\right)\right\|}{\left\|u_{\lambda_{n}}\right\|} \\
& \leq \frac{\beta\left[\int_{0}^{\omega} b(s) f\left(s, u\left(h_{2}(s)\right), \int_{-\zeta}^{0} e(s) u(s+v) d v\right) d s+\sum_{0 \leq t_{k} \leq \omega} I_{k}\left(t_{k}, u_{\lambda_{n}}\left(t_{k}\right)\right)\right]}{\left\|u_{\lambda_{n}}\right\|} \\
& \leq \frac{\beta\left[\gamma \mathbf{M}+p \mathbf{M}^{*}\right]}{\left\|u_{\lambda_{n}}\right\|} \\
& \leq \frac{2 \beta\left[\gamma \mathbf{M}+p \mathbf{M}^{*}\right]}{\eta} \quad(n>\mathbf{N}),
\end{aligned}
$$

which contradicts $\lambda_{n} \rightarrow 0^{+}$.

If $\eta=0$, then $\left\|u_{\lambda_{n}}\right\| \rightarrow 0$ for sufficiently large $n(n>\mathbf{N})$, and therefore it follows from $\left(\mathrm{H}_{5}\right)$ that for any $\varepsilon>0$ there exists $r_{3}>0$ such that

$$
f(t, x, y) \leq \varepsilon x, \quad I_{k}(t, x) \leq \varepsilon \mathcal{X}, \quad \forall 0 \leq x, y \leq r_{3},
$$


and hence it follows from (2.10) that

$$
\begin{aligned}
\frac{1}{\lambda_{n}} & =\frac{\left\|\int_{t}^{t+\omega} G(t, s) b(s) f\left(s, u\left(h_{2}(s)\right), \int_{-\zeta}^{0} e(s) u(s+v) d v\right) d s+\sum_{t \leq t_{k} \leq t+\omega} G\left(t, t_{k}\right) I_{k}\left(t_{k}, u_{\lambda_{n}}\left(t_{k}\right)\right)\right\|}{\left\|u_{\lambda_{n}}\right\|} \\
& \leq \frac{\beta\left[\int_{0}^{\omega} b(s) f\left(s, u\left(h_{2}(s)\right), \int_{-\zeta}^{0} e(s) u(s+v) d v\right) d s+\sum_{0 \leq t_{k} \leq \omega} I_{k}\left(t_{k}, u_{\lambda_{n}}\left(t_{k}\right)\right)\right]}{\left\|u_{\lambda_{n}}\right\|} \\
& \leq \frac{\beta\left[\gamma \varepsilon\left\|u_{\lambda_{n}}\right\|+p \varepsilon\left\|u_{\lambda_{n}}\right\|\right]}{\left\|u_{\lambda_{n}}\right\|} \\
& =\beta(\gamma+p) \varepsilon .
\end{aligned}
$$

Since $\varepsilon$ is arbitrary, we have $\lambda_{n} \rightarrow+\infty(n \rightarrow+\infty)$ in contradiction with $\lambda_{n} \rightarrow 0^{+}$. Therefore, $\left\|u_{\lambda}\right\| \rightarrow+\infty$ as $\lambda \rightarrow 0^{+}$and the proof is complete.

\section{Existence under (1.3) and (1.4)}

Firstly, let us denote that for each fixed constant $\rho>0$,

$$
\begin{aligned}
& h^{*}(\rho):=\max \{g(s) \mid 0 \leq s \leq \rho\}, \\
& h_{*}(\rho):=\min \{g(s) \mid 0 \leq s \leq \rho\} .
\end{aligned}
$$

Theorem 4.1 Assume that $\left(\mathrm{H}_{1}\right)-\left(\mathrm{H}_{4}\right)$ hold and gatisfies (1.3).

$\left(\mathrm{H}_{7}\right)$ If $f^{0}=0$ and $I^{0}(k)=0, k=0,1, \ldots, p-1$, then there exist $\lambda_{\rho}>0$ and $\mu_{\rho}>0$ such that problem (1.1) has a positive periodic solution $u$ with

$$
\sup _{t \in J} u(t) \leq \rho \quad \text { for } \lambda>\lambda_{\rho} \text { and } \mu>\mu_{\rho}
$$

$\left(\mathrm{H}_{8}\right)$ If $f_{0}=+\infty$ and $I_{0}(k)=+\infty, k=0,1, \ldots, p-1$, such that problem (1.1) has a positive periodic solution $u$ with

$$
\sup _{t \in J} u(t) \leq \rho \quad \text { for } 0<\lambda<\lambda_{\rho} \text { and } 0<\mu<\mu_{\rho}
$$

Corollary 4.1 Assume that $\left(\mathrm{H}_{1}\right)-\left(\mathrm{H}_{4}\right)$ hold and the function $g$ satisfies (1.3).

(i) If $f^{0}=0$ and $I^{0}(k)=0, k=0,1, \ldots, p-1$, then there exists $\lambda_{\rho}>0$ or $\mu_{\rho}>0$ such that problem (1.1) has a positive periodic solution $u(t)$ with property (4.3).

(ii) If $f_{0}=+\infty$ or $I_{0}(k)=+\infty, k=0,1, \ldots, p-1$, then there exist $\bar{\lambda}_{\rho}>0$ and $\bar{\mu}_{\rho}>0$ such that problem (1.1) has a positive periodic solution $u(t), t \in J$ with property (4.4).

Let $\rho$ be the constant in Theorem 4.1. Define a cone $K[\rho]$ in $E$ by

$$
K[\rho]=\left\{u \in E: u(t) \geq \frac{h^{*}(\rho)\left(1-h_{*}(\rho)\right)}{1-h^{*}(\rho)}\|u\|\right\} .
$$

For every $\iota>0$, let

$$
\Omega_{\iota}=\{u \in K[R]:\|u\|<\iota\}, \quad \partial \Omega_{\iota}=\{u \in K[R]:\|u\|=\iota\}
$$


and

$$
\bar{\Omega}_{\iota}=\{u \in K[R]:\|u\| \leq \iota\} .
$$

Define an operator $T_{\lambda}: E \rightarrow E$ by

$$
\begin{aligned}
(T u)(t)= & \lambda \int_{t}^{t+\omega} G_{u}(t, s) b(s) f\left(s, u\left(h_{2}(s)\right), \int_{-\zeta}^{0} e(s) u(s+v) d v\right) d s \\
& +\mu \sum_{t \leq t_{k}<t+\omega} G_{u}\left(t, t_{k}\right) I_{k}\left(t_{k}, u\left(t_{k}\right)\right),
\end{aligned}
$$

where

$$
G_{u}(t, s)=\frac{e^{\int_{t}^{s} a(v) g\left(u\left(h_{1}(v)\right)\right) d v}}{e^{\int_{0}^{\omega} a(v) g\left(u\left(h_{1}(v)\right)\right) d v}-1}, \quad s \in[t, t+\omega]
$$

It follows from (4.1) and (4.2) that

$$
h_{*}(\rho) \leq g(u) \leq h^{*}(\rho), \quad u \in \bar{\Omega}_{\rho},
$$

and hence, for $u \in \bar{\Omega}_{\rho}$, we have

$$
\frac{1}{\sigma^{h^{*}(\rho)}-1}=\alpha(\rho) \leq G_{u}(t, s) \leq \beta(\rho)=\frac{\sigma^{h_{*}(\rho)}}{\sigma^{h_{*}(\rho)}-1}, \quad s \in[t, t+\omega],
$$

where $\sigma$ is defined in (2.8).

Similarly, for $0<r<\rho$, we can define

$$
h^{*}(r):=\max \{g(s) \mid 0 \leq s \leq r\}, \quad h_{*}(r):=\min \{g(s) \mid 0 \leq s \leq r\} .
$$

Obviously,

$$
h_{*}(r) \leq g(u) \leq h^{*}(r)
$$

and, for $u \in \bar{\Omega}_{r}$, we have

$$
\begin{aligned}
\frac{\sigma^{h^{*}(\rho)}}{1-\sigma^{h^{*}(\rho)}} & \leq \frac{\sigma^{h^{*}(r)}}{1-\sigma^{h^{*}(r)}} \leq G_{u}(t, s) \\
& \leq \frac{1}{1-\sigma^{h_{*}(r)}} \leq \frac{1}{1-\sigma^{h_{*}(\rho)}}, \quad s \in[t, t+\omega], u \in \bar{\Omega}_{r} .
\end{aligned}
$$

Lemma 4.1 (See [43]) Assume that $\left(\mathrm{H}_{1}\right)-\left(\mathrm{H}_{4}\right)$ hold. Let $\rho>0$ be fixed. Then for each $r$ : $0<r \leq \rho$, we have $T\left(\bar{\Omega}_{r}\right) \subset K[\rho]$ and $T: \bar{\Omega}_{r} \rightarrow K[\rho]$ is compact and continuous.

Remark 4.1 (See [43]) Notice that for the cone $K[\rho]$ defined by (4.3) we cannot obtain

$$
T(K[\rho]) \subset K[\rho]
$$


However, Lemma 4.1 shows that, for every $r \in(0, \rho]$,

$$
T\left(\bar{\Omega}_{r}\right) \subset K[\rho]
$$

which is enough for us to apply the fixed point theorem in cones.

Lemma 4.2 (See [43]) Assume that $\left(\mathrm{H}_{1}\right)-\left(\mathrm{H}_{4}\right)$ hold. If $u$ is a fixed point of $T$ in $K[\rho]$, then $u$ is a periodic solution of problem (1.1).

Proof of Theorem 4.1 Let $R=\rho$ and $\alpha(\rho)$ replace $\alpha, \beta(\rho)$ replace $\beta$ in the proof of Theorem 2.2(i) and (ii) and repeat the argument of Theorem 2.2(i) and (ii). Then we can prove that Theorem 4.1 holds.

Remark 4.2 Let the condition (1.4) replace (1.3) in Theorem 4.1, the results of Theorem 4.1 also hold.

Remark 4.3 Since the function $G_{u}(t, s)$ is related to $u$ and the operator $T$ does not satisfy

$$
T(K[\rho]) \subset K[\rho]
$$

we have the following facts.

(i) $u \rightarrow 0$ means there exists a sufficiently small positive number $r$ such that $u \in[0, r]$. So one can easily choose a positive number $\rho$ to control $r$.

(ii) $u \rightarrow \infty$ means there exists a sufficiently large positive number $R$ such that $u \in[R, \infty]$, which implies that it is very difficult to find a positive number $\eta$ to control $R$.

Therefore, we only prove the existence of positive periodic solutions for problem (1.1) under (1.3) and $f$ and $I_{k}(k=0,1, \ldots, p-1)$ satisfying one of the following conditions:

(i) $f^{0}=0$ and $I^{0}(k)=0, k=0,1, \ldots, p-1$;

(ii) $f_{0}=\infty$ and $I_{0}(k)=\infty, k=0,1, \ldots, p-1$.

However, we do not give any information on the existence of positive periodic solutions for problem (1.1) in the four following cases:

(iii) (1.3) holds, $f^{\infty}=0$ and $I^{\infty}(k)=0, k=0,1, \ldots, p-1$;

(iv) (1.3) holds, $f_{\infty}=\infty$ and $I_{\infty}(k)=\infty, k=0,1, \ldots, p-1$;

(v) (1.4) holds, $f^{\infty}=0$ and $I^{\infty}(k)=0, k=0,1, \ldots, p-1$;

(vi) (1.4) holds, $f_{\infty}=\infty$ and $I_{\infty}(k)=\infty, k=0,1, \ldots, p-1$.

This and the proof of Theorem 3.1 imply the following.

Remark 4.4 If (1.3) or (1.4) hold, then we cannot obtain the parameter dependence results of positive periodic solution for problem (1.1).

\section{An example}

To illustrate how our main results can be used in practice, we present an example. Let

$$
p=1, \quad e(t) \equiv \frac{1}{\xi}, \quad \omega=2 \pi, \quad r=1 .
$$


Example 5.1 Consider the following equation:

$$
\left\{\begin{aligned}
u^{\prime}(t)= & -a(t) g\left(u\left(h_{1}(t)\right)\right) u(t) \\
& +\lambda b(t) f\left(t, u\left(h_{2}(t)\right), \int_{-\zeta}^{0} e(s) u(t+s) d s\right), \quad t \in \mathbf{R}, t \neq t_{k}, \\
u\left(t_{k}^{+}\right)- & u\left(t_{k}\right)=\mu I_{k}\left(t_{k}, u\left(t_{k}\right)\right), \quad k \in \mathbf{Z},
\end{aligned}\right.
$$

where $\lambda>0$ and $\mu>0$ are parameters, and

$$
\begin{aligned}
& a(t)=\frac{1}{2 \pi}, \quad b(t)=1-\sin t, \quad g\left(u\left(h_{1}(t)\right)\right)=\frac{1}{3}+e^{-u(t)}, \\
& I_{k}\left(t_{k}, u\left(t_{k}\right)\right)=\left(\frac{3}{2}+\sin \left(t_{k}\right)\right) u\left(t_{k}\right), \quad k \in \mathbf{Z}, \\
& f\left(t, u\left(h_{2}(t)\right), \int_{-\zeta}^{0} e(s) u(t+s) d s\right)=\left(\frac{1}{2}+\frac{1}{3} \sin t\right) u(t)+\int_{-\zeta}^{0} \frac{1}{\zeta} u(t+s) d s
\end{aligned}
$$

Conclusion 5.1 For any $\lambda>\frac{3 e^{\frac{4}{3}}\left(e^{\frac{4}{3}}-1\right)^{2}}{14 \pi\left(e^{\frac{1}{3}}-1\right)}$ and $\mu>\frac{e^{\frac{4}{3}}\left(e^{\frac{4}{3}}-1\right)^{2}}{3\left(e^{\frac{1}{3}}-1\right)}$, problem (5.1) has a positive $2 \pi$ periodic solution.

Proof It follows from (5.3) that $\left(\mathrm{H}_{1}\right)-\left(\mathrm{H}_{4}\right)$ of Theorem 2.1 hold. Noticing that $p=1$, we have

$$
[0, \omega] \cap\left[t_{k}, k \in \mathbf{Z}\right]=\left\{t_{0}\right\} .
$$

Letting $t_{0}=\pi$, then

$$
\frac{1}{3} \leq g(u)=\frac{1}{3}+e^{-u} \leq \frac{4}{3}, \quad f_{\infty}=\frac{7}{6}<\frac{11}{6}=f^{\infty}, \quad I_{\infty}=I^{\infty}=\frac{3}{2}
$$

So we can take

$$
l=\frac{1}{3}, \quad L=\frac{4}{3} .
$$

On the other hand, it follows from (5.1) that

$$
\begin{aligned}
& \sigma=e^{\int_{0}^{\omega} a(t) d t}=e, \quad \alpha=\frac{1}{\sigma^{L}-1}=\frac{1}{e^{\frac{4}{3}}-1}, \\
& \beta=\frac{\sigma^{L}}{\sigma^{l}-1}=\frac{e^{\frac{4}{3}}}{e^{\frac{1}{3}}-1}, \quad \delta=\frac{\sigma^{l}-1}{\sigma^{L}\left(\sigma^{L}-1\right)}=\frac{e^{\frac{1}{3}}-1}{e^{\frac{4}{3}}\left(e^{\frac{4}{3}}-1\right)}, \\
& \gamma=\int_{0}^{2 \pi}(1-\sin t) d t=2 \pi, \quad m_{r}=\frac{7\left(e^{\frac{1}{3}}-1\right)}{6 e^{\frac{4}{3}}\left(e^{\frac{4}{3}}-1\right)}, \quad m^{*}=\frac{3\left(e^{\frac{1}{3}}-1\right)}{2 e^{\frac{4}{3}}\left(e^{\frac{4}{3}}-1\right)}
\end{aligned}
$$

and

$$
\begin{aligned}
& \lambda_{0}=\left(2 \alpha m_{r} \gamma\right)^{-1} r=\frac{3 e^{\frac{4}{3}}\left(e^{\frac{4}{3}}-1\right)^{2}}{14 \pi\left(e^{\frac{1}{3}}-1\right)}, \\
& \mu^{0}=\left(2 \alpha m^{*} p\right)^{-1} r=\frac{e^{\frac{4}{3}}\left(e^{\frac{4}{3}}-1\right)^{2}}{3\left(e^{\frac{1}{3}}-1\right)} .
\end{aligned}
$$




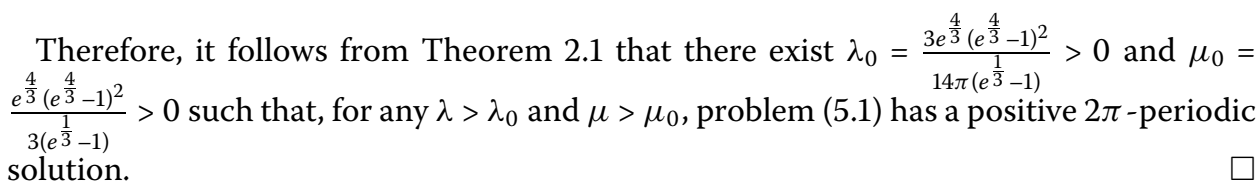

\section{Competing interests}

The authors declare that they have no competing interests.

\section{Authors' contributions}

XZ completed the main study and carried out the results of this article. MF checked the proofs and verified the calculation. All authors read and approved the final manuscript.

\section{Author details}

'Department of Mathematics and Physics, North China Electric Power University, Beijing, 102206, People's Republic of China. ${ }^{2}$ School of Applied Science, Beijing Information Science \& Technology University, Beijing, 100192, People's Republic of China.

\section{Acknowledgements}

This work is sponsored by the project NSFC (11301178), the Fundamental Research Funds for the Central Universities (2014ZZD10, 2014MS58) and the Scientific Research Common Program of Beijing Municipal Commission of Education (KM201511232018). The authors are grateful to the anonymous referees for their constructive comments and suggestions, which have greatly improved this paper.

Received: 26 January 2015 Accepted: 24 July 2015 Published online: 11 August 2015

\section{References}

1. Kuang, Y: Delay Differential Equations: With Applications in Population Dynamics. Academic Press, Boston (1993)

2. Yan, J: Existence and global attractivity of positive periodic solution for an impulsive Lasota-Wazewska model. J. Math. Anal. Appl. 279, 111-120 (2003)

3. Gyöi, I, Ladas, G: Oscillation Theory of Delay Differential Equations: With Applications. Clarendon, Oxford (1991)

4. Lalli, BS, Zhang, B: On a periodic delay population model. Q. Appl. Math. 52, 35-42 (1994)

5. Gopalsamy, K, Kulenović, MRS, Ladas, G: Environmental periodicity and time delays in a food-limited population model. J. Math. Anal. Appl. 147, 545-555 (1990)

6. Cushing, JM: Periodic time-dependent predator-prey system. SIAM J. Appl. Math. 32, 82-95 (1977)

7. Chow, SN: Existence of periodic solutions of autonomous functional differential equations. J. Differ. Equ. 15, 350-378 (1974)

8. Freedman, HI, Wu, J: Periodic solutions of single-species models with periodic delay. SIAM J. Math. Anal. 23, 689-701 (1992)

9. Liu, X, Li, W: Existence and uniqueness of positive periodic solutions of functional differential equations. J. Math. Anal. Appl. 293, 28-39 (2004)

10. Wang, H: Positive periodic solutions of functional differential equations. J. Differ. Equ. 202, 354-366 (2004)

11. Sun, $\mathrm{X}, \mathrm{Huo}, \mathrm{H}, \mathrm{Ma}, \mathrm{C}$ : Periodic solutions of a class of impulsive neutral delay differential equation. Appl. Math. Comput. 219, 3947-3955 (2012)

12. Cheng, S, Zhang, G: Existence of positive periodic solutions for non-autonomous functional differential equations. Electron. J. Differ. Equ. 2001, 59 (2001)

13. Jiang, D, Wei, J: Existence of positive periodic solutions of nonautonomous functional differential equations. Chin. Ann. Math. 20, 715-720 (1999) (in Chinese)

14. Wan, A, Jiang, D: Existence of positive periodic solutions for functional differential equations. Kyushu J. Math. 56, 193-202 (2002)

15. Chu, J, Nieto, JJ: Impulsive periodic solutions of first-order singular differential equations. Bull. Lond. Math. Soc. 40 143-150 (2008)

16. Li, J, Nieto, JJ, Shen, J: Impulsive periodic boundary value problems of first-order differential equations. J. Math. Anal. Appl. 325, 226-236 (2007)

17. Yan, J, Shen, J: Impulsive stabilization of functional differential equations by Lyapunov-Razumikhin functions. Nonlinear Anal. 37, 245-255 (1999)

18. Li, J, Shen, J: New comparison results for impulsive functional differential equations. Appl. Math. Lett. 23, 487-493 (2010)

19. Yang, X, Shen, J: Nonlinear boundary value problems for first-order impulsive functional differential equations. Appl. Math. Comput. 189, 1943-1952 (2007)

20. Liu, Y: Periodic boundary value problems for first order functional differential equations with impulse. J. Comput. Appl. Math. 223, 27-39 (2009)

21. Liu, Y: Further results on periodic boundary value problems for nonlinear first-order impulsive functional differential equations. J. Math. Anal. Appl. 327, 435-452 (2007)

22. $\mathrm{He}, \mathrm{Z}, \mathrm{Yu}, \mathrm{J}$ : Periodic boundary value problem for first-order impulsive functional differential equations. J. Math. Anal. Appl. 272, 67-78 (2002)

23. Ding, $\mathrm{W}, \mathrm{Han}, \mathrm{M}, \mathrm{Mi}$, J: Periodic boundary value problem for the second-order impulsive functional differential equations. Comput. Math. Appl. 50, 491-507 (2005)

24. Chen, L, Sun, J: Nonlinear boundary value problem of first-order impulsive functional differential equations. J. Math. Anal. Appl. 318, 726-741 (2006) 
25. Liu, Y, Ge, W: Stability theorems and existence results for periodic solutions of nonlinear impulsive delay differential equations with variable coefficients. Nonlinear Anal. 57, 363-399 (2004)

26. Luo, Z: Multiple positive periodic solutions for functional differential equations with impulses and a parameter. Abstr. Appl. Anal. (2014). doi:10.1155/2014/812867

27. Luo, Z, Luo, L, Zeng, Y: Positive periodic solutions for impulsive functional differential equations with infinite delay and two parameters. J. Appl. Math. (2014). doi:10.1155/2014/751612

28. Yan, J: Existence of positive periodic solutions of impulsive functional differential equations with two parameters. J. Math. Anal. Appl. 327, 854-868 (2007)

29. Cooke, KL, Kaplan, JL: A periodicity threshold theorem for epidemic and population growth. Math. Biosci. 31, 87-104 (1976)

30. Cooke, KL, Yorke, JA: Some equations modelling growth processes and gonorrhea. Math. Biosci. 16, 75-101 (1973)

31. Mackey, MC, Glass, L: Oscillations and chaos in physiological control systems. Science 197, $287-289$ (1997)

32. Jin, Z, Wang, H: A note on positive periodic solutions of delayed differential equations. Appl. Math. Lett. 23, 581-584 (2010)

33. Ye, D, Fan, M, Wang, H: Periodic solutions for scalar functional differential equations. Nonlinear Anal. 62, 1157-1181 (2005)

34. Li, Y, Fan, X, Zhao, L: Positive periodic solutions of functional differential equations with impulses and a parameter. Comput. Math. Appl. 56, 2556-2560 (2008)

35. Graef, JR, Kong, L: Periodic solutions of first order functional differential equations. Appl. Math. Lett. 24, 1981-1985 (2011)

36. Graef, JR, Kong, L: Existence of multiple periodic solutions for first order functional differential equations. Math. Comput. Model. 54, 2962-2968 (2011)

37. Franco, D, Liz, E, Torres, PJ: Existence of periodic solutions for functional equations with periodic delay. Indian J. Pure Appl. Math. 38, 143-152 (2007)

38. Wu, J, Wang, Z: Positive periodic solutions of second-order nonlinear differential systems with two parameters. Comput. Math. Appl. 56, $43-54$ (2008)

39. Padhi, S, Srivastava, S: Multiple periodic solutions for a nonlinear first order functional differential equations with applications to population dynamics. Appl. Math. Comput. 203, 1-6 (2008)

40. Olach, R: Positive periodic solutions of delay differential equations. Appl. Math. Lett. 26, 1141-1145 (2013)

41. Amster, $P$, Idels, L: Periodic solutions in general scalar non-autonomous models with delays. Nonlinear Differ. Equ. Appl. 20, 1577-1596 (2013)

42. Chen, R, Ma, R, He, Z: Positive periodic solutions of first-order singular systems. Appl. Math. Comput. 218, $11421-11428(2012)$

43. Ma, R, Chen, R, Chen, T: Existence of positive periodic solutions of nonlinear first-order delayed differential equations. J. Math. Anal. Appl. 384, 527-535 (2011)

44. Weng, A, Sun, J: Positive periodic solutions of first-order functional differential equations with parameter. J. Comput. Appl. Math. 229, 327-332 (2009)

45. Guo, DJ, Lakshmikantham, V: Nonlinear Problems in Abstract Cones. Academic Press, New York (1988)

\section{Submit your manuscript to a SpringerOpen ${ }^{\ominus}$ journal and benefit from:}

- Convenient online submission

Rigorous peer review

Immediate publication on acceptance

- Open access: articles freely available online

- High visibility within the field

- Retaining the copyright to your article 